\title{
HOW HIGH THE TEMPERATURE OF A LIQUID BE RAISED WITHOUT BOILING?
}

\author{
${ }^{\ddagger}$ Mala Das, B. K. Chatterjee ${ }^{\star}$, B. Roy and S. C. Roy ${ }^{\dagger}$ \\ ${ }^{\ddagger}$ Department of Physics, Bose Institute \\ $\ddagger 93 / 1$ A. P. C. Road, Calcutta 700009, India
}

${ }^{\ddagger}$ Lines to be deleted from the manuscript for blindfold refereeing

\begin{abstract}
How high a temperature of a liquid be raised beyond its boiling point without vaporizing (known as the limit of superheat) is an interesting subject of investigation. A new method of finding the limit of superheat of liquids is presented here. The superheated liquids are taken in the form of drops suspended in a dust free gel. The temperature of the superheated liquid is increased very slowly from room temperature to the temperature at which the liquid nucleates to boiling. The nucleation is detected acoustically by a sensitive piezo-electric transducer, coupled to a multi channel scaler (MCS) and the nucleation rate is observed as a function of time. The limit of superheat measured by the present method supersedes other measurements and theoretical predictions in reaching the temperature closest to the critical temperature of the liquids.
\end{abstract}

PACS numbers: 64.60.-i, 64, 64.70.Fx, 64.60.Qb, 64.60.My 
Any fluid that exists in the liquid form above its boiling temperature is said to be superheated. These liquids are in a metastable state in the thermodynamic sense and can be nucleated to form vapor by homogeneous nucleation or by the presence of heterogeneous nucleation sites such as gas pockets, vapor bubbles, solid impurities etc. or by the radiation interactions caused by charged particles, neutrons etc. Vapor embryos of different sizes, which are responsible for homogeneous nucleation, are produced at thermal equilibrium in the superheated liquid. The superheated state owes its existence to an energy barrier which causes the vapor embryo to collapse, rather than lead to nucleation, if it is less than a critical size.

A liquid can not be superheated up to the critical temperature, there is a limit to the maximum attainable temperature for any given liquid without boiling. This limit is called the 'limit of superheat of the liquid' $\left(\mathrm{T}_{s l}\right)$, where the height of the energy barrier which maintains the superheated state is of the order of $\mathrm{kT}$ and this temperature is a characteristic of any liquid. In addition to its importance in basic science, the knowledge of $\mathrm{T}_{s l}$ is important in a number of industrial operations where a hot, nonvolatile liquid comes in contact with a cold volatile liquid. If the temperature of the hot liquid reaches to the limit of superheat of the cold liquid, explosive boiling would result. This explosive boiling is a potential hazard in damaging equipment and injure personnel in the vicinity of the blast [1]. The study of $\mathrm{T}_{s l}$ has another importance since the discovery of bubble chamber by Glaser[2] and superheated drop detector[3]. The operation of this detector depends on the degree of superheat of the 
liquid, more the liquid is superheated more sensitive is the detector to lower energy radiations[4]. The minimum energy detectable by such detector is therefore limited by the limit of superheat of the detecting liquid. The limit of superheat of liquids can be estimated from the theory and can be measured experimentally. Theoretical calculations are performed either from the pure thermodynamic considerations or using the statistical mechanics. Very good and comprehensive reviews on homogeneous nucleation of lquid and on the limit of superheat are available in literature $[5,6,7]$. One has to note that theoretical calculations are performed for 'pure' homogeneous nucleation where the chance of heterogeneous nucleation arising out of various interfaces with different surface energies e.g. gas-liquid, liquid-liquid, solid-gas etc. is completely excluded.

Experimental results reported so far are far below the critical temperature of the liquids. One of the reasons being that observing 'pure' homogeneous nucleation experimentally, without any chance of heterogeneous nucleation is difficult to achieve. Hence, the goal is to reduce the chance of heterogeneous nucleation as far as possible and to use an improved method of quantitative detection of nucleation to see how close one can reach experimentally to the predicted limit of superheat. The present experiment is designed to achieve this goal. Superheated sample used in this investigation is a homogeneous suspension of superheated drops of three liquids (R-12 : $\mathrm{CCl}_{2} F_{2}$, R-114: $\mathrm{C}_{2} \mathrm{Cl}_{2} F_{4}$ and R-22: $\left.\mathrm{CHCl} F_{2}\right)$ in a dust free, visco-elastic, degassed gel medium. Suspending the superheated liquid in another liquid (gel) reduces the 
chance of heterogeneous nucleation. Nucleation is detected acoustically by a piezoelectric transducer[8] and the pulses thus received are digitized and recorded as a function of time by a multichannel scaler. This improved method of determining $\mathrm{T}_{s l}$ supersedes all other measured values in reaching closest to the critical temperatures. Reviews on previous experimental techniques of measuring the limit of superheat of liquid have been described in detail by Avedisian[6]. As has been found from this literature, all previous experiments except one rely on the qualitative observation of the nucleation visually and therefore the present measurement constitutes the first quantitative measurement of $\mathrm{T}_{s l}$ using digital electronics.

The limit of superheat can be estimated either from the thermodynamic stability theory or from the analysis of the dynamics of formation of the critical sized vapor embryos (statistical mechanical theory). The superheated state of a liquid is a metastable state and the limit of this metastable state is represented on the P-V diagram by the spinodal curves. For a pure liquid, the spinodal curve or the thermodynamic limit of superheat is defined by states for which

$$
\left(\frac{d P}{d V}\right)_{T}=0
$$

Temperley[9] calculated the value of maximum superheat temperature using van der Waals' equation of state. The maximum limit of superheat of a given liquid can be expressed as

$$
t_{m}=\frac{27 T_{c}}{32}
$$


where $t_{m}$ is the limit of superheat of the liquid. For mathematical simplicity this has been calculated by considering the ambient pressure to be zero. At atmospheric pressure i.e. at $\mathrm{P}=1, \mathrm{t}_{m}$ will be slightly greater than the corresponding value at $\mathrm{P}=0$. Other equations of state such as modified Bertholet equation and RedlichKwong equation have also been used to calculate the limit of superheat[5]. As has been observed by Blander and Katz[5], experimental values of thermodynamic limit clearly exceeded the Van der Waals limit at least for five liquids.

For most of the organic liquids the thermodynamic limit of superheat can be represented empirically[1] by

$$
T_{s l}=T_{c}\left[0.11\left(P / P_{c}\right)+0.89\right]
$$

where $\mathrm{T}_{c}$ is the critical temperature, $\mathrm{P}_{c}$ is the critical pressure and $\mathrm{P}$ is the ambient pressure.

Another method of estimating $\mathrm{T}_{s l}$ using statistical mechanics involves considerations of the rate processes of nucleation to form vapor embryos in a superheated liquid. This method does not yield an absolute value of $\mathrm{T}_{s l}$ but it allows one to estimate the probable rate of formation of critical-sized vapor embryos in a superheated liquid at a given temperature. If the rate is very low within the time scale of the experiment, one considers no nucleation would occur, while if the rate is very high, then one assumes that $\mathrm{T}_{s l}$ has been reached. The rate of homogeneous nucleation 
$(\mathrm{J})$ as given approximately by the Volumer-Doring formula is given by[1]

$$
J=N f \exp -\left(\frac{B}{k T}\right)
$$

where $\mathrm{J}$ is the expected rate of formation of critical sized vapor embryos per unit volume, $\mathrm{f}$ is a frequency factor which in general is of the order of $10^{11} \mathrm{sec}^{-1}, \mathrm{~N}$ is the number density of molecules in the superheated liquid and B is the minimum amount of energy needed to form a vapor bubble of critical size as given by Gibbs[10] from reversible thermodynamics is

$$
B=16 \pi \gamma^{3}(T) / 3\left(p_{v}-p_{o}\right)^{2}
$$

where $\gamma(T)$ is the liquid-vapor interfacial tension, $P_{v}$ is vapor pressure of the superheated liquid and $P_{o}$ is the ambient pressure. It is to be noted in this connection that which value of $\mathrm{J}$ is proper to calculate $\mathrm{T}_{s l}$ is not defined and therefore one has to make some 'judicious choice' of a rate which would correspond to $\mathrm{T}_{s l}$. A J value of $10^{6}$ nucleation $/ \mathrm{cm}^{3}$.sec is often used to define the limit of superheat temperature.

It is to be noted that all the above discussions are related with the classical theory of nucleation. Effect of other factors like diffusion, viscosity and other hydrodynamical constraints are discussed by Blander and Katz [5]. As has been pointed out by them, contributions arising out of these effects in calculating $\mathrm{T}_{s l}$ of pure liquids are not very significant.

The experiment is carried out with superheated liquids of R12 (b.p. $-29.79{ }^{\circ} \mathrm{C}$ ), R114 (b.p. $3.6^{\circ} \mathrm{C}$ ) and R22 (b.p. $-40.5^{\circ} \mathrm{C}$ ). The superheated drops are suspended 
in dust free, de-gassed visco-elastic gel. The gel is a mixture of 'aquasonic' gel available commercially and glycerine. A glass vial containing the superheated drops homogeneously suspended in gel is placed on the top of a thin layer of degassed gel taken in a beaker. The gel in the beaker improves the acoustic coupling between the superheated drops in the vial and the transducer. The beaker is placed on a piezoelectric transducer with a coupling gel. Some pure gel is placed on the top of the sample and a thermometer was inserted in the pure gel so as to avoid any contact with the superheated liquid sample, thus reducing the chance of heterogeneous nucleation from the liquid-glass interface. The nucleation in superheated drops is detected by the transducer, the output of the transducer is digitized and recorded by a multi channel scaler. The vial was wrapped with a heating coil covering the gel and sample. The temperature of the sample is increased slowly from room temperature and the count rate $(\mathrm{dN} / \mathrm{dt})$ is recorded in MCS. As nucleation proceeds, the number of superheated drops are depleted and hence the nucleation rate is normalized with respect to the number of drops present. What we expect ideally is $\left(\frac{1}{N}\right) \frac{d N}{d t}$ is zero till the temperature reaches the limit of superheat where there will be a sudden increase in $\left(\frac{1}{N}\right) \frac{d N}{d t}$ (entire liquid nucleates) and will be no nucleation beyond this temperature. Considering the experimental uncertainty, one may observe the similar behavior as presented in Fig. 1. The comparison of observed limit of superheat with other experimental results is presented in the table below. The reduced limit of superheat defined as $T_{s l} / T_{c}$ (taken in ${ }^{\circ} \mathrm{K}$ ) for these liquids is also presented in the table along with theoretically 
predicted values and other experimental results.

\begin{tabular}{|c|c|c|c|c|c|c|c|c|}
\hline \multirow{3}{*}{ Liquid } & \multirow{3}{*}{$\begin{array}{l}\mathrm{T}_{c} \\
{ }^{o} K\end{array}$} & \multicolumn{2}{|c|}{ observed $\mathrm{T}_{s l}{ }^{\circ} \mathrm{C}$} & \multicolumn{5}{|c|}{ Reduced limit of superheat $\left[\mathrm{T}_{s l}(\mathrm{~K}) / \mathrm{T}_{c}(\mathrm{~K})\right]$} \\
\hline & & \multirow[b]{2}{*}{ Present } & \multirow[b]{2}{*}{ Others } & \multicolumn{3}{|c|}{ Predicted values from } & \multicolumn{2}{|c|}{ Experiment } \\
\hline & & & & (eqn.2) & (eqn.3) & (eqn.4) & Present & Others. \\
\hline R12 & 384.5 & 80.0 & $72.0[1]$ & 0.84 & 0.89 & 0.90 & 0.92 & $0.90[1]$ \\
\hline R114 & 418.7 & 120.5 & $102.0[1]$ & 0.84 & 0.89 & 0.91 & 0.94 & $0.90[1]$ \\
\hline $\mathrm{R} 22$ & 369.0 & 57.5 & $54.0[1]$ & 0.84 & 0.89 & 0.89 & 0.89 & $0.89[1]$ \\
\hline & & & & & & & & $0.89[6]$ \\
\hline
\end{tabular}

As could be seen from the table, the measured limit of superheat exceeded the predicted limit of superheat and other experimental values. It is to be noted in this connection that all theretical predictions are approximate as discussed before. Therefore the present experimental measurements indicate the need of improved calculation of limit of superheat. That the Van der Waals' limit is exceeded was reported before by Blander and Katz[5]. This table also gives an useful insight about the nucleation process. As can be seen from the table, for liquids with lower boiling points it is harder to reach closer to the critical temperature. This is quite expected as the chances of heterogeneous nucleation increases in case of liquids with lower boiling points. Whether complete elimination of heterogeneous nucleation in experimental 
measurement is possible or not is an open question. No other measurement have been able to reach so close to the critical temperature. It is to be noted in this connection that the limit of superheat of only 14 liquids out of 56 liquids studied by Blander and Katz [5] hardly exceeded $90 \%$ of the critical temperature.

Therefore, by reducing the chances of heterogeneous nucleation by suspending the superheated sample in another 'pure' liquid and using precise electronic measurement we have been able to reach closer to the critical temperature hitherto unattainable. Inspite of the fact that theoretical calculations are performed for 'pure' homogeneous nucleation, they fall below the experimental values indicate the inadequacy of the present method of calculation discussed here and warrants improved calculations.

$\ddagger\left[{ }^{\star}\right.$ Present address : Chemistry Dept. Univ. of Utah. Salt Lake City. USA.]

$\ddagger{ }^{\dagger}$ Author for correspondence : Fax no. 9133 350-6790,

email : scroy@bosemain.boseinst.ernet.in]

\section{${ }^{\ddagger}$ Lines to be deleted from the manuscript for blindfold refereeing}

1. R. C. Reid Advances in Chemical Engineering 12, 199 (1983).

2. D. A. Glaser Phys. Rev 8, 665 (1952).

3. R. E. Apfel US Patent 4,143,274 (1979).

4. R.E. Apfel, S. C. Roy and Y.C. Lo Phys. Rev. A. 31, 3194 (1985).

5. Blander M. and Katz J. L. AIChE 21, 833 (1975).

6. Avedisian C. T. J. Phys. Chem. Data 14, 695, (1985). 
7. Basu D. K. and Sinha D. B. Ind. J. Phys. 42, 198, (1968).

8. Apfel R. E. and Roy S. C. Rev. Sci. Inst. 54, 1397, (1983).

9. Temperley H. N. V. Proc. Phys. Soc. 59, 199, (1947).

10. Gibbs J. W. Translations of the Connecticut Academy III, p.108 (1875). 

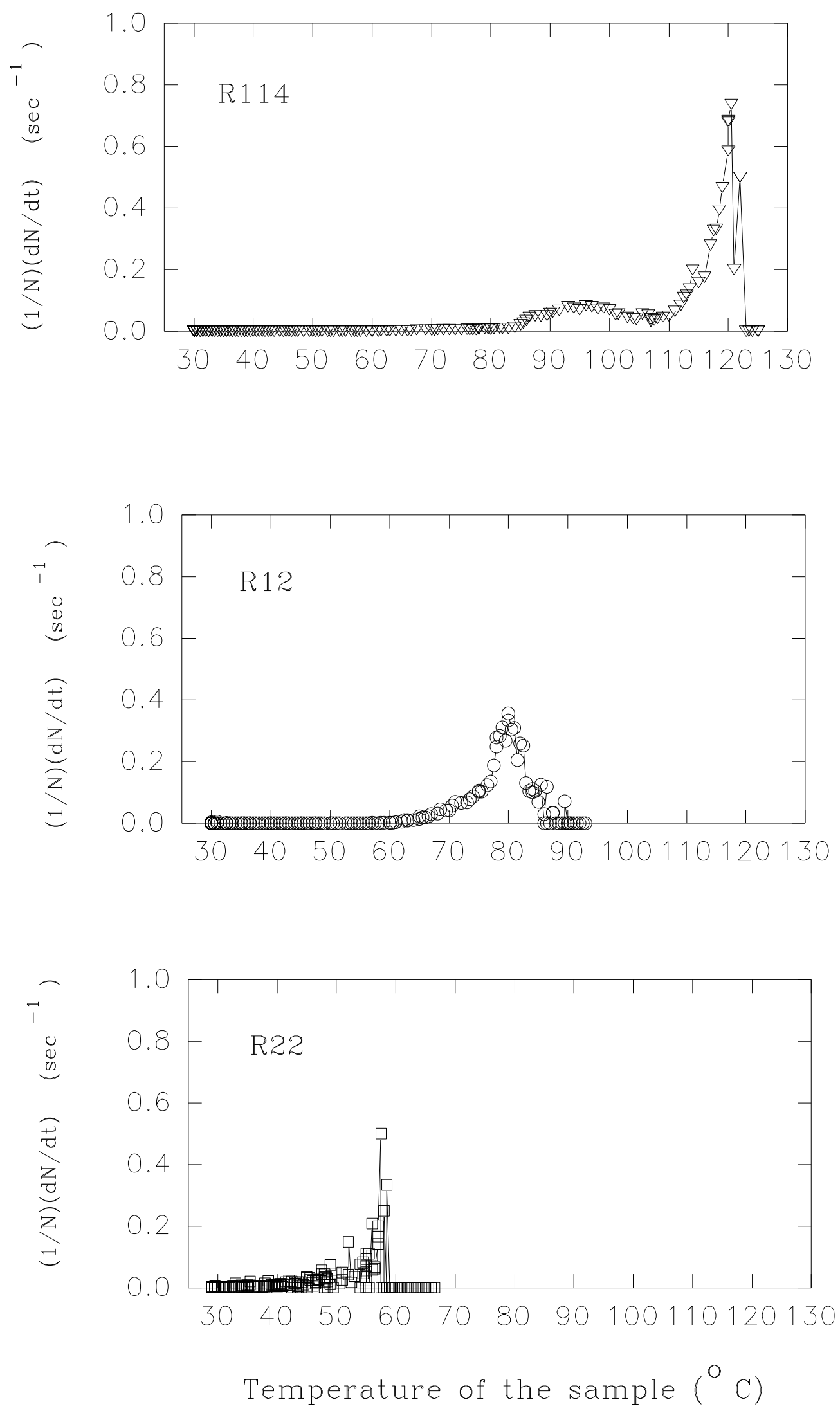

Fig.1 Variation of nucleation rate with the temperature of the sample 